\title{
Localized Shape Variations for Classifying Wall Motion in Echocardiograms
}

\author{
K.Y. Esther Leung and Johan G. Bosch \\ Biomedical Engineering, Thoraxcenter, Erasmus MC, Rotterdam, The Netherlands \\ k.leung@erasmusmc.nl \\ http://www . erasmusmc.nl/ThoraxcenterBME
}

\begin{abstract}
To quantitatively predict coronary artery diseases, automated analysis may be preferred to current visual assessment of left ventricular (LV) wall motion. In this paper, a novel automated classification method is presented which uses shape models with localized variations. These sparse shape models were built from four-chamber and two-chamber echocardiographic sequences using principal component analysis and orthomax rotations. The resulting shape parameters were then used to classify local wall-motion abnormalities of LV segments. Various orthomax criteria were investigated. In all cases, higher classification correctness was achieved using significantly less shape parameters than before rotation. Since pathologies are typically spatially localized, many medical applications involving local classification should benefit from orthomax parameterizations.
\end{abstract}

\section{Introduction}

Coronary artery diseases are a major cause of death in the western world. Detection of wall-motion abnormalities of the left ventricle (LV), widely accepted as predictors for these diseases, is therefore of great clinical importance. To obtain quantitative measures of LV wall motion, automated analysis of LV wall motion may be preferred to currently visual, therefore qualitative, assessments. The goal of this study is to evaluate a new automated classification approach for detecting local wall-motion abnormalities. The method uses point-distribution models with localized variations obtained with orthomax rotations.

Point-distribution models, or shape models, are parametric representations of a set of shapes. These models have been used extensively in various medical image processing contexts, especially segmentation [1, 2]. Shape models are often built using Principal Component Analysis (PCA), which maximizes the variance of the input data. This results in models with global variations. Previously, PCA shape models of LV endocardial borders were used to classify global clinical parameters (e.g. LV volume) as well as local parameters (e.g. wall motion of LV segments) 3. However, relatively many shape modes were needed to classify the local wall motion, because global shape parameters were used. We therefore hypothesize that models with local variations should be able to represent local wall motion using less shape modes.

Several methods for building more localized shape models have been proposed, including independent component analysis [4] and various sparse PCA methods 
[5], 6]. Recently, Stegmann et al. 7] suggested a method using orthomax rotations, which seems particularly attractive due to its computational feasibility in high-dimensional spaces. The applicability for localized classification was mentioned in that paper, but has not yet been investigated.

To determine whether localized shape models can improve classification of local wall-motion abnormalities of the left ventricle, shape models were constructed using PCA and rotated according to the orthomax criterion. The varimax, quartimax, and factor-parsimony criteria were investigated. Classification correctness, the number of shape modes needed, and cluster representation were studied in the original and rotated shape space, for different proportions of retained variance.

\section{Methods}

\subsection{Shape Modeling}

Shape models of the LV endocardial contours were constructed using full-cycle $2 \mathrm{D}+$ time $(2 \mathrm{D}+\mathrm{T})$ echocardiograms. By modeling the complete cardiac cycle, typical motion patterns associated with certain pathologies were included. More details of the model can be found in our previous work [8].

Each $2 \mathrm{D}+\mathrm{T}$ shape was represented as a vector $\mathbf{x}$, consisting of 37 equally distributed landmark coordinates per phase. Sixteen cardiac phases were used for each shape. Shape models describing the main variations in a patient population were built using PCA: $\mathbf{x}=\overline{\mathbf{x}}+\mathbf{\Phi} \mathbf{b}$, where $\overline{\mathbf{x}}$ denotes the average shape, $\boldsymbol{\Phi}=$ $\left(\phi_{1}|\ldots| \phi_{p}\right)$ the $n \times p$ matrix of orthogonal shape eigenvectors or modes, and $\mathbf{b}$ a vector of shape coefficients. Any new shape can be approximated in the PCA space, spanned by the $p$ orthogonal eigenvectors, using the pseudoinverse $\left(\boldsymbol{\Phi}^{-1}\right)$ of the eigenvector matrix $\left(\mathbf{b} \approx \boldsymbol{\Phi}^{-1}(\mathbf{x}-\overline{\mathbf{x}})\right)$.

To obtain a more compact model, only a proportion $f$ of the total variance $V=\sum_{i=1}^{p} \lambda_{i}$ was retained: $\sum_{i=1}^{k} \lambda_{i} \geq f V$, where $k$ denoted the number of eigenvectors with the largest eigenvalues $\lambda_{i}$.

\subsection{Orthomax Rotations}

Orthomax rotations were applied to the PCA shape models to produce models with localized spatial variations [7, 9]. Orthomax rotations are reparameterizations of the PCA space producing a simple basis. The orthogonal orthomax rotation matrix $\mathbf{R}$ is calculated by maximizing the criterion $\xi$ :

$$
\xi=\left\{\sum_{j=1}^{k} \sum_{i=1}^{n} G_{i j}^{4}-\frac{\gamma}{n} \sum_{j=1}^{k}\left[\sum_{i=1}^{n} G_{i j}^{2}\right]^{2}\right\} / n,
$$

where $G_{i j}$ denotes the scalar element in the $i^{\text {th }}$ row and $j^{\text {th }}$ column in the rotated eigenvector matrix $\mathbf{G}=\mathbf{\Phi R}$, and $\gamma$ is determined by the orthomax type. The shape coefficients after rotation $\mathbf{b}_{R}$ can be found with $\mathbf{b}_{R}=\mathbf{R}^{-1} \mathbf{b}$. 


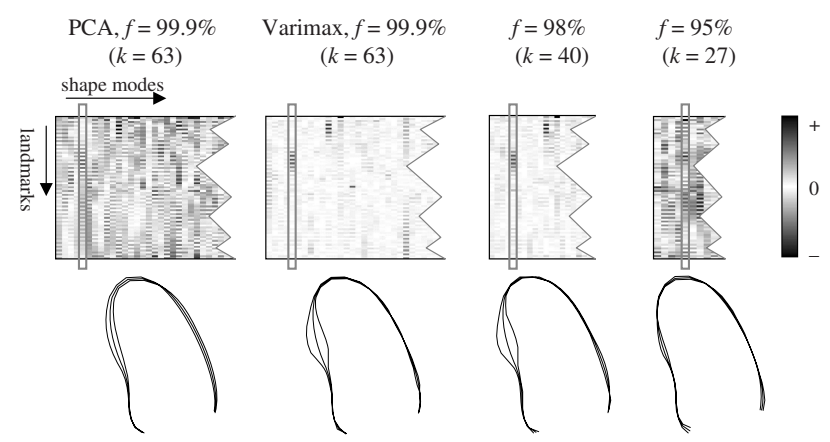

Fig. 1. Top row: eigenvector matrices of PCA and varimax-rotated shape model of the four-chamber. Bottom row: end-diastolic shape variations of the fifth mode, showing more localization for shape models with higher proportions of retained variance $f$.

Interestingly, the orthogonal orthomax criterion is equivalent to the CrawfordFerguson criterion, which is a weighted sum of row and column complexity of the eigenvector matrix [10]. Therefore, orthomax rotations can be interpreted as a redistribution of the factor loadings of the eigenvector matrix so that each row or column has a minimal number of nonzero elements, i.e. columns or rows are as sparse as possible. The two extremes are quartimax $(\gamma=0)$ and factor parsimony $(\gamma=n)$, favoring row sparsity and column sparsity respectively [9]. Varimax $(\gamma=1)$, a commonly-used type, resides somewhere in between [1]. When applied to a shape eigenvector matrix, the variation is emphasized in certain modes and spatial regions, whereas the variation is suppressed in other regions (see Fig. 11). In practice, complete row or column sparsity cannot be achieved because the shape model is restricted to the observed, physically allowed variations in the training shapes.

An important property to consider is the number of shape modes $k$. PCA modes with high eigenvalues describe global variation, whereas modes with low eigenvalues generally contain noise. Eliminating these noise modes before the orthomax rotation may lead to more representative local variations. However, if too many modes are removed, important information needed for classification may be lost. Also, a lower $k$ generally results in less localized shape variations, because each mode must capture more variations (see Fig.11). Therefore, different proportions of retained variance $f$ were investigated : $f=95 \%, 98 \%, 99 \%$ and $99.9 \%$ corresponding to $k=27,40,48$, and 63 modes in the four-chamber $(4 \mathrm{C})$ model, and to $k=25,38,46$, and 62 modes in the two-chamber $(2 \mathrm{C})$ model.

\subsection{Wall-Motion Classification}

Classification of wall-motion abnormalities was demonstrated on dobutamine stress echocardiography data from 129 unselected infarct patients [12. The total data set was split randomly into a training set (TRN) of 65 patients and a testing set (TST) of 64 patients. Endocardial contours were drawn in the apical $4 \mathrm{C}$ and 


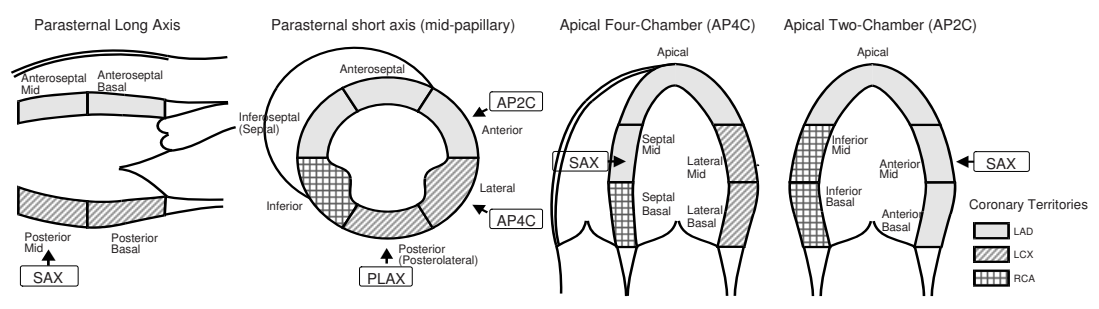

Fig. 2. 13-segment model of the left ventricle (LAD = left anterior descending artery, $\mathrm{LCX}=$ left circumflex artery, RCA = right coronary artery)

$2 \mathrm{C}$ sequences using a semi-automated technique based on pattern matching, dynamic programming, and spatiotemporal geometric models [8] $4 \mathrm{C}$ and $2 \mathrm{C}$ shape models were built with the image sequences in the TRN set [3. Shape coefficients $\mathbf{b}$ and $\mathbf{b}_{R}$ were calculated for all data sets and used as predictor (or independent) variables in the classification.

Myocardial wall motion in these images was evaluated visually by consensus of two expert readers, independently of the shape models. Motion scores were assigned to each of the $13 \mathrm{LV}$ segments $(0=$ normokinesia, $1=$ hypokinesia, 2 $=$ akinesia, $3=$ dyskinesia; see Fig. 2). Wall-motion scores of each of the nine segments in the $4 \mathrm{C}$ and $2 \mathrm{C}$ views were grouped into a normal class (score = 0 ) and an abnormal (score $>0$ ) class and used as the response (dependent) variables in the classification.

Two classification experiments were performed, representing an 'ideal' and a 'worst-case' situation. In both cases, the shape model was built on the TRN set. In the 'ideal' case, a leave-one-out approach was used where the classifier was trained on the TRN set but one sample and tested with that sample. This process was then repeated for all TRN samples. In the 'worst-case' situation, the classifier was trained on the whole TRN set and then tested on all TST cases. This resembled classification in the real-world: both shape model and classifier were trained from a limited training set and tested on completely 'new' shapes.

To assess the classification in the PCA and orthomax rotated space, cluster measurements were computed. Common measures of cluster compactness and cluster separation are the within-class scatter matrix $\mathbf{S}_{W}$ and the between-class scatter matrix $\mathbf{S}_{B}\left[13\right.$. Since $\mathbf{S}_{W}$ and $\mathbf{S}_{B}$ are related to the variance of the point-cluster in each direction, a better measure of cluster quality is the ratio $J$ of the trace of the two scatter matrices $\left([14\right.$, p. 311$): J=\frac{\operatorname{tr}\left(\mathbf{S}_{B}\right)}{\operatorname{tr}\left(\mathbf{S}_{W}\right)}$.

\section{Results}

Orthomax rotations were applied to $4 \mathrm{C}$ and $2 \mathrm{C}$ shape models, using an iterative method based on singular value decomposition [7], as implemented in Matlab (v. 7.0.4 (R14), 2005). Whereas PCA shape modes are ordered according to variance, thus exhibiting global variations in the first modes, orthomax modes show local variations in most modes (see Fig. 33). 

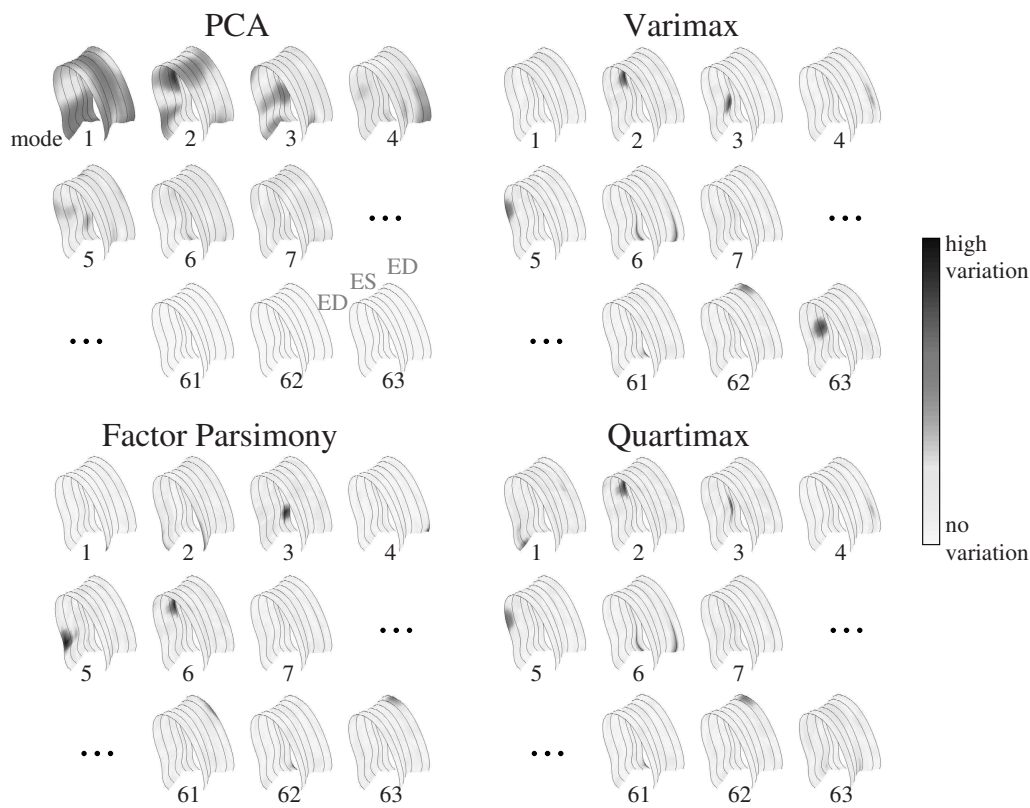

Fig. 3. Global PCA and localized orthomax-rotated modes of the $4 \mathrm{C}$ shape model, $f=99.9 \%$. Absolute displacements of the landmarks due to \pm 3 SD model-variation are shown on the average $2 \mathrm{D}+\mathrm{T}$ shape.

Shape parameters were used to predict the presence of wall motion abnormalities per LV segment. Classification was performed using the Linear Discriminant Analysis option in the statistical package SPSS (v. 11.0.1, 2001). Shape parameters were added using the 'stepwise' option, using the 'unexplained variance' criterion. Significantly less orthomax modes were needed than PCA modes, without compromising classification correctness (i.e. the proportion of segments correctly classified as normal or abnormal), regardless of the criterion used (Table 1). Also, models with higher $f$ needed less shape modes during classification. The results per segment are given in Table 2 for the varimax criterion.

Fig. 4. shows cluster quality $J$ (section 2.3) in PCA and varimax space. Clearly, classification in the varimax space gives larger $J$, i.e. better cluster separation, than in the PCA space in most cases.

\section{Discussion and Conclusions}

In this study, localized shape models of the left ventricle were generated using orthomax rotations. Using these localized shape models, significantly fewer parameters were needed for classifying segmental wall motion in 2D echocardiographic sequences, while preserving classification correctness. Best results were obtained using all PCA modes before rotations, probably as a result of a sparser rotated basis; apparently, the classification was not hampered by possible noise 
Table 1. Classification correctness versus the number of shape modes used (mean $\pm \mathrm{SD}$ ), for different proportions of retained variance $f$ in the shape models, and different orthomax criteria. ${ }^{*}$ denotes significantly $(p<0.05$, paired $t$-test) less shape parameters than PCA.

\begin{tabular}{lllll}
\hline & \multicolumn{3}{c}{ Classification correctness } & \#parameters \\
& $f$ & TRN L-1-O & TST &. \\
\hline \hline PCA & $99.9 \%$ & $88.9 \pm 5.9 \%$ & $74.0 \pm 9.4 \%$ & $8.0 \pm 3.0$ \\
\hline quartimax & $99.9 \%$ & $90.1 \pm 5.2 \%$ & $75.4 \pm 9.8 \%$ & $5.6 \pm 3.9^{*}$ \\
factor parsimony & $99.9 \%$ & $89.4 \pm 5.7 \%$ & $76.3 \pm 10.3 \%$ & $5.4 \pm 3.2^{*}$ \\
varimax & $99.9 \%$ & $91.1 \pm 4.5 \%$ & $76.5 \pm 10.5 \%$ & $5.1 \pm 3.2^{*}$ \\
& $99 \%$ & $88.9 \pm 5.9 \%$ & $76.0 \pm 8.7 \%$ & $5.7 \pm 3.3^{*}$ \\
& $98 \%$ & $87.7 \pm 7.0 \%$ & $75.8 \pm 9.5 \%$ & $6.4 \pm 3.5$ \\
& $95 \%$ & $86.3 \pm 6.1 \%$ & $76.3 \pm 9.0 \%$ & $6.6 \pm 3.7$ \\
\hline
\end{tabular}

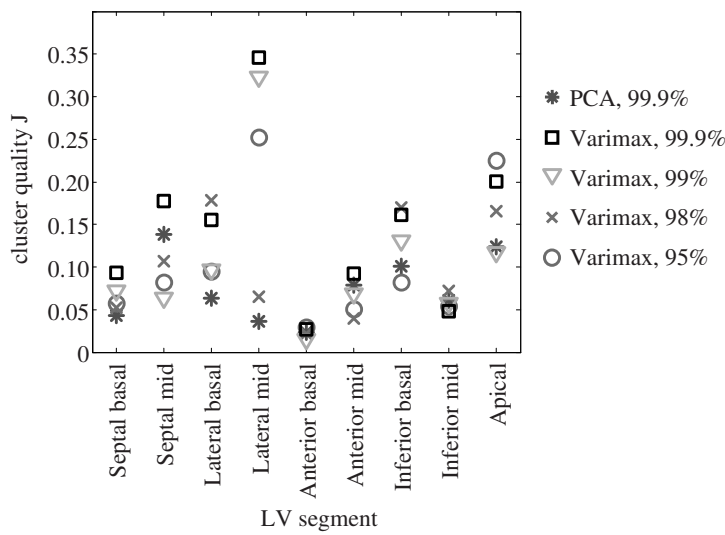

Fig. 4. Cluster quality $J$ (see section 2.3) shows better cluster separation in varimax than PCA classification space, for different proportions of retained variance

in the higher PCA modes. Similar results were obtained for the quartimax, varimax, and factor parsimony criteria, probably because the reparameterizations are restricted by the allowable variations in the training set. The relatively large improvement in the lateral region may be due to the combination of the orthomax rotation and the variations in the TRN set, which by chance produced a sparser parameter representation for those segments.

Furthermore, the normal and abnormal wall motion classes were better separated in the orthomax parameter space than in the original PCA space. A clear relationship between the improvement in classification correctness, the number of shape modes used, and cluster quality could not be determined because of the small number of data sets in this study, but generally speaking, better classification can be expected in a better-defined parameter space. The cluster measurements revealed that there was still a reasonable amount of overlap of the classes. This may be partly due to the high variability of visual wall motion 
Table 2. Classification correctness versus the number of PCA and varimax shape parameters used, for nine segments

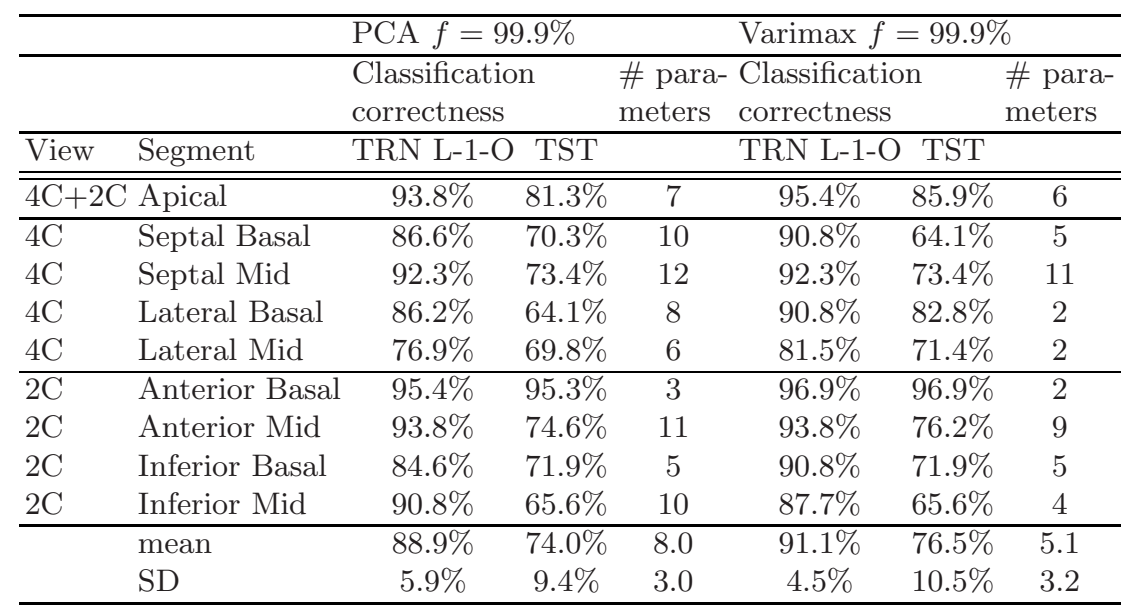

scoring. Regrettably, this variability could not be determined for this data set because the scoring was performed by consensus. In the future, we would like to use a more objective measure such as quantitative deformation parameters as the gold standard, rather than visual wall motion scores. The class overlap also suggests that better classification might be achieved with a nonlinear discriminant function, such as support vector machines.

While PCA modes are ordered according to variance, orthomax modes are naturally unordered. In future work, we would like to investigate categorization of modes more thoroughly, e.g. according to the locality of the shape variations 13. This might allow us to preselect the shape modes for classification, instead of having the classifier do the selection.

Moreover, it would be interesting to compare the proposed orthomax method with other localization methods, such as independent component analysis 4 and sparse PCA methods [5], 6]. Recently, Frouin et al. [15] proposed an intensitybased method for detecting wall-motion abnormalities using factor analysis, which might also be used for rotating shape models to a predefined motion pattern. This is a subject of further investigation.

Finally, since pathologies are typically spatially localized, we anticipate many medical applications where sparse representations are preferred to the conventional PCA approach. The orthomax criterion is shown to be suitable for building these sparse representations with relative ease. Although not explored in this study, sparse texture models can be constructed in a very similar manner 7]. The direct cardiac application would be to examine myocardial thickening, which might also be a predictor of coronary disease. In the future, we would like to extend the method to coupled shape models of rest and stress stage cardiac contours, to investigate local differences in wall-motion. 


\section{Acknowledgments}

Financial support from the Dutch Technology Foundation STW is gratefully acknowledged. We thank O. Kamp and F. Nijland for providing the patient data, G. van Burken for the data analysis support, and M. van Stralen, M. M. Voormolen, M. Sonka, and M. B. Stegmann for the useful discussions.

\section{References}

1. Cootes, T.F., Edwards, G.J., Taylor, C.J.: Active appearance models. IEEE Trans. Pattern Anal. Machine Intell. 23(6), 681-685 (2001)

2. Stegmann, M.B.: Generative interpretation of medical images. PhD thesis, Technical University of Denmark (2004)

3. Bosch, J.G., Nijland, F., Mitchell, S.C., Lelieveldt, B.P.F., Kamp, O., Reiber, J.H.C., Sonka, M.: Computer-aided diagnosis via model-based shape analysis: Automated classification of wall motion abnormalities in echocardiograms. Acad. Radiol. 12(3), 358-367 (2005)

4. Üzümcü, M., Frangi, A.F., Reiber, J.H.C., Lelieveldt, B.P.F.: Independent component analysis in statistical shape models. Proc. SPIE Med. Imag.: Image Processing 5032, 375-383 (2003)

5. Zou, H., Hastie, T., Tibshirani, R.: Sparse principal component analysis. tech. rep. Standford University (2004)

6. Sjöstrand, K., Stegmann, M.B., Larsen, R.: Sparse principal component analysis in medical shape modeling. SPIE Med. Imag.: Image Processing 6144, 61444X (2006)

7. Stegmann, M.B., Sjöstrand, K., Larsen, R.: Sparse modeling of landmark and texture variability using the orthomax criterion. SPIE Med. Imag.: Image Processing 6144, 61441G (2006)

8. Bosch, J.G., Mitchell, S.C., Lelieveldt, B.P.F., Nijland, F., Kamp, O., Sonka, M., Reiber, J.H.C.: Automatic segmentation of echocardiographic sequences by active appearance motion models. IEEE Trans. Med. Imag. 21(11), 1374-1383 (2002)

9. Browne, M.W.: An overview of analytic rotation in exploratory factor analysis. Multivar. Behav. Res. 36(1), 111-150 (2001)

10. Crawford, C.B., Ferguson, G.A.: A general rotation criterion and its use in orthogonal rotation. Psychometrika 35(3), 321-332 (1970)

11. Kaiser, H.F.: The varimax criterion for analytic rotation in factor analysis. Psychometrika 23(3), 187-200 (1958)

12. Nijland, F., Kamp, O., Verhorst, P.M.J., de Voogt, W.G., Bosch, H.G., Visser, C.A.: Myocardial viability: impact on left ventricular dilatation after acute myocardial infarction. Heart 87, 17-22 (2002)

13. Suinesiaputra, A., Frangi, A.F., Üzümcü, M., Reiber, J.H.C., Lelieveldt, B.P.F.: Extraction of myocardial contractility patterns from short-axis MR images using independent component analysis. In: Sonka, M., Kakadiaris, I.A., Kybic, J. (eds.) CVAMIA 2004. LNCS, vol. 3117, pp. 75-86. Springer, Heidelberg (2004)

14. Webb, A.R.: Statistical Pattern Recognition, 2nd edn. John Wiley \& Sons, Ltd. Chichester (2002)

15. Frouin, F., Delouche, A., Raffoul, H., Diebold, H., Abergel, E., Diebold, B.: Factor analysis of the left ventricle by echocardiography (falve): a new tool for detecting regional wall motion abnormalities. Eur. J. Echocardiogr. 5(5), 335-346 (2004) 